\title{
Approximants to the Tonks-Langmuir theory for a collisionless annular plasma
}

\author{
Yunchao Zhang," Christine Charles, and Rod Boswell \\ Space Plasma, Power and Propulsion Laboratory, Research School of Physics and Engineering, The Australian National University, \\ Bldg 60, Mills Road, ACT 2601, Australia
}

(Received 25 August 2015; published 4 December 2015)

\begin{abstract}
Maclaurin series approximant and Padé rational approximant are used to solve the Tonks-Langmuir theory for an annular plasma and investigate the radial transport behavior of charged particles. Coefficients of the well-known Maclaurin approximant are given in a novel form of recurrence relations which are convenient for computation and present a lower limit for the annular ratio of inner radius to outer radius (i.e., this approximant is not applicable to annular geometries with small inner radii). The newly introduced Padé approximant extrapolates the annular ratio limit determined by the Maclaurin approximant to a lower value and hence is applicable to most annular geometries. General radial profiles of the normalized plasma density and mean drift velocity of ions are given across the annulus and they are independent of the gas type and the Paschen number of the discharge. The annular modeling is applied to an argon plasma and obtains the electron temperature as a function of the Paschen number for different annular geometries.
\end{abstract}

DOI: 10.1103/PhysRevE.92.063103

PACS number(s): 52.25.-b, 52.27.Aj

\section{INTRODUCTION}

Tonks and Langmuir [1] reported a free-fall scenario of ions in a collisionless plasma in their classic paper "A General Theory of the Plasma of an Arc," where the newly created ions had a zero drift velocity and fell towards the plasma boundary without encountering any collisions. The electrons were assumed to be in equilibrium and governed by the Boltzmann relation. The Tonks-Langmuir theory was represented in the form of an integral equation referenced here as the "T-L integral equation." Their model has been widely used in studies of transport phenomena in low-temperature plasmas [2,3], especially for the collisionless low-pressure regime, together with the Schottky model [4] for the collisional high-pressure regime and the Godyak model [5] for the collisional intermediate-pressure regime. The T-L integral equation was originally solved using the plausible Maclaurin series approximation for the plane-parallel and cylindrical plasmas [1]. An analytical-form solution could also be given in terms of Dawson functions, but it was limited to the plane-parallel geometry [6].

The annular geometry is a natural extension for the cylindrical geometry when an inner object is inserted, e.g., a probe within a plasma column $[1,7]$ or an inner quartz tube in a plasma source [8-10]. A solution to collisional annular plasmas has been reported in our previous study [11] where the introduction of an effective ion temperature unified the Schottky model and Godyak model. However, to date the Tonks-Langmuir model has not been fully investigated for a collisionless annular plasma. Some preliminary discussion for the annular case was indeed given by Tonks and Langmuir in their original work [1], but it was incomplete and only restricted to the case of uniform ion generation. It should be noted that their form of the Maclaurin approximant for an annular plasma was actually incomplete due to the lack of even-index terms which could be omitted for the cylindrical and plane-parallel geometries but not for the annulus.

\footnotetext{
*yunchao.zhang@anu.edu.au
}

This study focuses on the single-component, electronpositive-ion, low-temperature plasmas where the ion thermal effect is neglected (cold ions). The electron temperature is assumed to be a constant across the plasma and hence the Boltzmann relation can be used to connect the plasma density and potential. Two cases of ion generation are included: (i) the ion generation density (the number of ions generated per second per unit volume) being proportional to the plasma density, defined as the P-case, and (ii) the ion generation density being uniform and independent of the plasma density, defined as the U-case. The P-case is mainly for the plasma dominated by one-step ionization $[1,12]$ while the multistep ionization [12,13] is beyond the scope of this paper and not presently investigated. The U-case normally occurs when the ion generation is supplied by an external ion source [12] or the diffusion effect [2] from an unconsidered dimension (e.g., the axial dimension is not included in this study). The ion loss mechanism is considered to be dominated by the plasma flux out of the boundary, and the volume recombination is negligible for the plasma of interest here which is a low-pressure electropositive discharge [2,3].

In Sec. II the T-L integral equation is introduced to describe the radial transport of charged particles in collisionless annular plasmas for the P- and U-cases. In order to solve this integral equation, Secs. III and IV present detailed mathematical analyses of two approximant methods: the Maclaurin series approximant and the Padé rational approximant. In Sec. III a complete Maclaurin approximant is given for the T-L integral equation of an annular plasma and, to the best of our knowledge, this is the first study to present the results of serial coefficients in forms of recurrence relations which are quite convenient for computational purpose. As the Maclaurin approximant is shown to be valid only for annular geometries with a relatively large annular ratio (defined as the ratio value of inner radius to outer radius), Sec IV introduces the Padé approximant $[14,15]$ to extend the applicable range of annular ratios to smaller values. In Sec. V the accuracy and validity of these two approximant methods are shown by substituting the related solution into the T-L integral equation to check the determined magnitude of the error, and the advantage of 
the Padé approximant in convergence is also verified for small annular ratios. The modeling results calculated from these two approximants are given in Sec. VI: First, radial profiles of the normalized plasma density and mean drift velocity of ions are obtained for different annular geometries and shown to be general results independent of the gas type and of the Paschen number. Subsequently, the model is applied to an annular plasma in argon to determine the electron temperature as a function of the Paschen number.

\section{T-L INTEGRAL EQUATION}

The Tonks-Langmuir theory assumes the electrons being in Maxwellian equilibrium and governed by the Boltzmann relation. The ion dynamics is determined from particle balance between ion generation and free-fall ion flux towards the plasma boundary, and this kinetic process can be represented by the T-L integral equation which is directly given in cylindrical coordinate without repeating the deduction details [1]:

$$
-s e^{-\eta} \pm \int_{s_{p}}^{s} s_{z} e^{-c \eta_{z}}\left(\eta-\eta_{z}\right)^{-\frac{1}{2}} d s_{z}=0,
$$

which connects two important dimensionless parameters that describe the transport performance of ions: the dimensionless potential (hence the normalized plasma density using the Boltzmann relation) given by $\eta=-\frac{\phi}{T_{e}}$, where $\phi$ and $T_{e}$ are the plasma potential and electron temperature, and the dimensionless position $s=\frac{r v_{z}^{(P / U)}}{\sqrt{2} u_{B}}$, where $r, v_{i z}$, and $u_{B}=$ $\sqrt{\frac{e T_{e}}{m_{i}}}\left(e\right.$ and $m_{i}$ represent the electron charge and ion mass) are the physical radial position, ion generation rate, and Bohm velocity, respectively. The superscript $(P / U)$ of the ion generation rate represents two cases [1]:

(i) P-case: $v_{i z}$ is the ionization rate and a constant for Maxwellian electrons in this study;

(ii) U-case: $v_{i z}$ is given by the ratio of the ion generation density (the number of ions generated per second per unit volume) to the maximum plasma density.

Solution to the T-L integral equation (1) is in the form of the dimensionless potential $\eta$ as a function of the dimensionless position $s$, i.e., $\eta=\eta(s)$. It can be seen from Eq. (1) that the solution depends on the dimensionless peak position $s_{p}$ which locates the maximum plasma density (corresponding to a zero plasma potential) across the annulus, and $c=1$ or 0 corresponds to the $\mathrm{P}$-case or the $\mathrm{U}$-case. Notation \pm represents the outer radial range beyond $s_{p}$ satisfying $s>s_{p}$ and the inner range within $s_{p}$ satisfying $s<s_{p}$, which will be used through the following paper. When $s_{p}=0$, the annulus is reduced to a normal cylindrical geometry.

Equation (1) is a nonlinear Volterra equation of the second kind for the unknown function $\eta(s)$. It has a kernel whose singularity is determined along $\eta_{z}=\eta$, and no solution for Volterra equations with this type of singular kernel can be found in the literature. Considering the monotonicity of $\eta(s)$ in the range of $s>s_{p}$ or $s<s_{p}$ where the plasma potential and radial position hold an injection relation, it is possible to cancel the kernel's singularity by transforming the above equation of $\eta(s)$ to a modified equation of the inverse of $\eta(s)$, i.e., $s(\eta)$, which yields:

$$
-s e^{-\eta} \pm \int_{0}^{\eta} s_{z} e^{-c \eta_{z}} \frac{d s_{z}}{d \eta_{z}} d\left[-2\left(\eta-\eta_{z}\right)^{\frac{1}{2}}\right]=0,
$$

where the singularity is removed from the integrand by variable substitution but a derivative term $\frac{d s_{z}}{d \eta_{z}}$ has to be introduced. Equation (2) is further modified by using the relative position $x=s-s_{p}$, and the square root of the dimensionless potential $\rho=\eta^{\frac{1}{2}}$ and $\rho_{z}=\rho \sin (\theta)$ to give [1]:

$$
-\left(s_{p}+x\right) e^{-\rho^{2}} \pm \int_{0}^{\frac{\pi}{2}}\left(s_{p}+x_{z}\right) e^{-c \rho_{z}^{2}} \frac{d x_{z}}{d \rho_{z}} d \theta=0
$$

which becomes an integral equation of $x(\rho)$, equivalent to $\eta(s)$ of Eq. (1), and satisfies $x=0$ when $\rho=0$, i.e., zero plasma potential is defined at the position of maximum plasma density. As $s_{p}$ is the only variable for the T-L integral equation, for each specific $s_{p}$ there is a corresponding "solution curve" $x(\rho)$ which is used to calculate plasma parameters such as the radial profiles of the normalized plasma density and the normalized mean drift velocity of ions (given in Sec. VI).

The range of solution curve $x(\rho)$ is determined by the inner boundary $s_{a}$ and outer boundary $s_{b}$ of an annular plasma. In order to keep a good consistency with Tonks and Langmuir's work, the plasma boundaries are defined as the position with an infinite electric field strength, i.e., $\frac{d \eta}{d s}=\infty$, which is equivalent to $\frac{d x}{d \rho}=0$, though this condition is normally untrue in most actual physical systems (one scenario where it is true could be a double layer boundary [16]). Other boundary conditions can be adapted by setting related constraint equations, e.g., a Bohm-type boundary is located by finding the position where the mean drift velocity is equal to the Bohm velocity $[2,3]$. The following two sections use approximant methods, the Maclaurin approximant and the Padé approximant, to give the solution curve $x(\rho)$ of the modified T-L integral equation (3).

\section{MACLAURIN SERIES APPROXIMANT}

As most smooth functions can be well approximated using the Taylor series (reduced to the Maclaurin series when it centers at zero) [17], the unknown function $x(\rho)$ is approximated by a Maclaurin series as follows:

$$
x=\sum_{i=0}^{+\infty} a_{i} \rho^{i} .
$$

The coefficients $\left\{a_{n}\right\}$ are derived by substituting the power series into the T-L integral equation (3) and omitting the rearranged coefficient of each power order of the equation. The zeroth element $a_{0}$ is always zero as $x=0$ when $\rho=0$. The first two elements $a_{1}$ and $a_{2}$ are same for both the P- and $\mathrm{U}$-cases and given by:

$$
a_{1}= \pm \frac{2}{\pi}, \quad a_{2}=\frac{ \pm a_{1}-a_{1}^{2}}{2 s_{p}} .
$$


The even-index terms $\left\{a_{2 n}\right\}$ are given in forms of recurrence relations for the $\mathrm{P}$-case:

$$
\begin{aligned}
a_{2 n}= & \frac{1}{(2 n) s_{p}}\left\{s_{p} \sum_{i=1}^{n-1} \frac{(-1)^{n-1-i}}{(n-i) !}(2 i) a_{2 i}\right. \\
& \pm \frac{(2 n-1) ! !}{(2 n-2) ! !} \sum_{i=1}^{n} \frac{(-1)^{n-i}}{(n-i) !} a_{2 i-1} \\
& \left.-\sum_{i=1}^{2 n-1} i a_{i} \sum_{j=1}^{n+1-\left\lceil\frac{i+1}{2}\right\rceil} \frac{(-1)^{n+1-\left\lceil\frac{i+1}{2}\right\rceil-j}}{\left(n+1-\left\lceil\frac{i+1}{2}\right\rceil-j\right) !} a_{2 j+2\left\lceil\frac{i-1}{2}\right\rceil-i}\right\}
\end{aligned}
$$

and the U-case:

$$
\begin{aligned}
a_{2 n}= & \frac{1}{(2 n) s_{p}}\left\{ \pm \frac{(2 n-1) ! !}{(2 n-2) ! !} \sum_{i=1}^{n} \frac{(-1)^{n-i}}{(n-i) !} a_{2 i-1}\right. \\
& \left.-\sum_{i=1}^{2 n-1} i a_{i} a_{2 n-i}\right\},
\end{aligned}
$$

where \lceil\rceil and $L\rfloor$ represent the ceiling and floor algorithms, respectively.

The odd-index terms $\left\{a_{2 n+1}\right\}$ for the P-case are given by:

$$
\begin{aligned}
a_{2 n+1}= & \frac{1}{(2 n+1) s_{p}}\left\{s _ { p } \left[ \pm \frac{(-2)^{n}}{(2 n-1) ! !} \frac{2}{\pi}\right.\right. \\
& \left.+\sum_{i=1}^{n} \frac{(-1)^{n-i}}{(n-i+1) !}(2 i-1) a_{2 i-1}\right] \\
& \pm \frac{(2 n) ! !}{(2 n-1) ! !} \frac{2}{\pi} \sum_{i=1}^{n} \frac{(-1)^{n-i}}{(n-i) !} a_{2 i} \\
& -\sum_{i=1}^{2 n} i a_{i} \quad \sum_{j=1}^{n+1-\left\lfloor\frac{i+1}{2}\right\rfloor} \frac{(-1)^{n+1-\left\lfloor\frac{i+1}{2}\right\rfloor-j}}{\left(n+1-\left\lfloor\frac{i+1}{2}\right\rfloor-j\right) !} \\
& \left.\times a_{2 j+2\left\lfloor\frac{i+1}{2}\right\rfloor-i-1}\right\}
\end{aligned}
$$

and the U-case:

$$
\begin{aligned}
a_{2 n+1}= & \frac{1}{(2 n+1) s_{p}}\left\{ \pm \frac{(2 n) ! !}{(2 n-1) ! !} \frac{2}{\pi}\left[s_{p} \frac{(-1)^{n}}{n !}\right.\right. \\
& \left.\left.+\sum_{i=1}^{n} \frac{(-1)^{n-i}}{(n-i) !} a_{2 i}\right]-\sum_{i=1}^{2 n} i a_{i} a_{2 n+1-i}\right\} .
\end{aligned}
$$

The coefficient series $\left\{a_{n}\right\}$ of the above Maclaurin series shows two important properties. First, those coefficient sequences for the radial ranges of $s>s_{p}$ and $s<s_{p}$, denoted as $\left\{a_{n}^{+}\right\}$and $\left\{a_{n}^{-}\right\}$, satisfy the relations:

$$
a_{2 n}^{+}=a_{2 n}^{-}, \quad a_{2 n+1}^{+}=-a_{2 n+1}^{-},
$$

which are identical for both the P- and U-cases and can be easily verified from the recurrence formulas (6) to (9). Hence $\left\{a_{n}^{+}\right\}$and $\left\{a_{n}^{-}\right\}$exhibit the same convergence or divergence performance which is determined from the modulus series

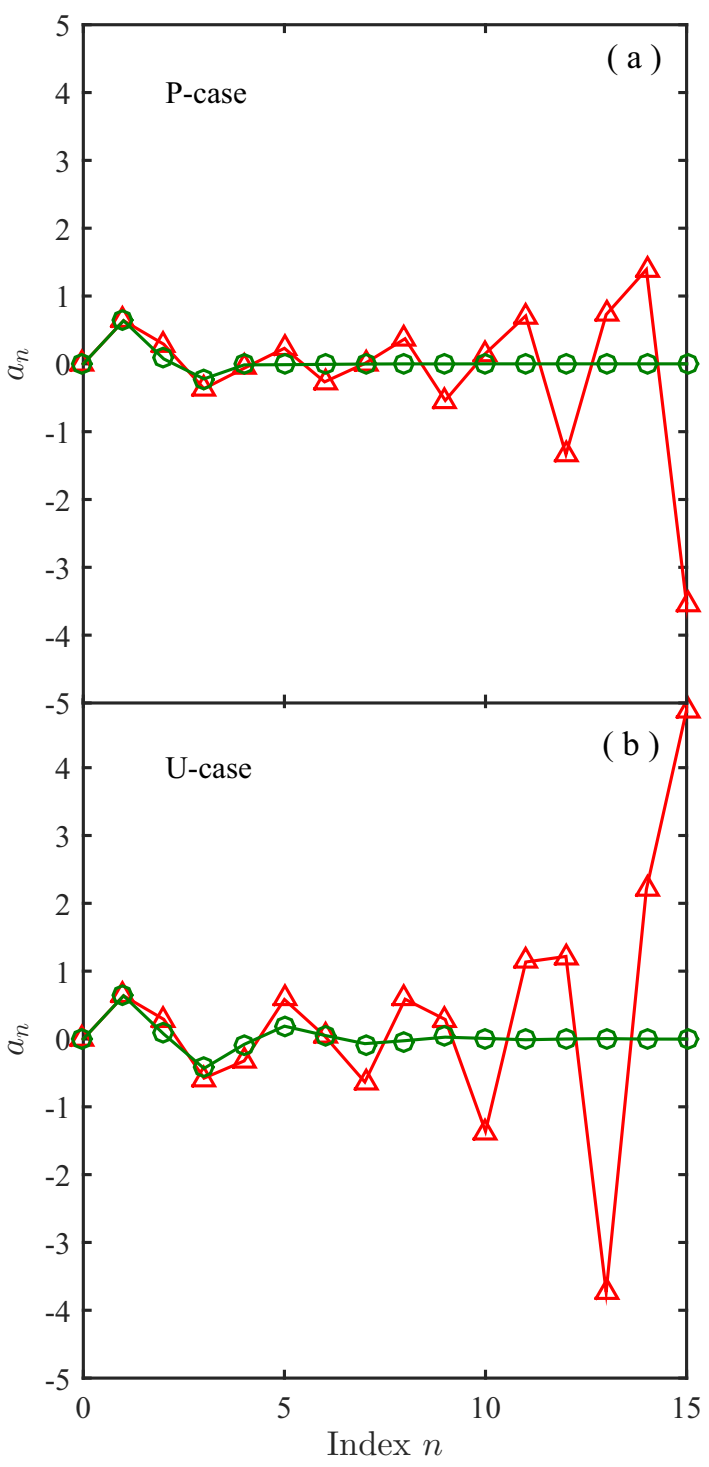

FIG. 1. (Color online) Coefficient series $\left\{a_{n}\right\}$ of the Maclaurin approximant for (a) the P-case and (b) the U-case with $s_{p}=0.4(\triangle)$ and $1.6(\bigcirc)$.

$\left\{\left|a_{n}\right|\right\}$. Following these notations, the outer and inner boundary locations $\left(s_{b}\right.$ and $\left.s_{a}\right)$ are determined from:

$$
\frac{d x^{ \pm}}{d \rho}=\sum_{i=1}^{\infty} i a_{n}^{ \pm} \rho^{i-1}=0,
$$

where $x$ has to be represented in the truncated form of the Maclaurin series (4), i.e., the Maclaurin series approximant, for a real computation with finite bits. This equation yields the solution of the square root of the dimensionless potential $\rho_{b}$ and $\rho_{a}$ at respective outer and inner boundaries, and they are used to calculate the boundary locations $s_{b}=x\left(\rho_{b}\right)+s_{p}$ and $s_{a}=x\left(\rho_{a}\right)+s_{p}$.

Second, the recurrence relations (6) to (9) show that $\left\{a_{n}\right\}$ is a function of the dimensionless peak position $s_{p}$ of the plasma density in the annulus, and the computational results suggest that $\left\{a_{n}\right\}$ is only convergent for large values of $s_{p}$ for which some typical values are listed in Fig. 1 as an illustration. 
The results of $\left\{a_{n}^{+}\right\}$with $s_{p}=1.6$ for the P-case $\left[\left\{a_{n}^{-}\right\}\right.$can be found using relations (10) and not plotted], represented by open circles in Fig. 1(a), present a convergent sequence. The open triangles for coefficient values with $s_{p}=0.4$ show a divergent oscillating behavior. The results for the U-case are given in Fig. 1(b) and show a similar performance. The divergent characteristics of $\left\{a_{n}\right\}$ for a small $s_{p}$ is clearly seen in the two panes. The convergence of $\left\{a_{n}\right\}$ has been found being satisfied when the value of $s_{p}$ is above a lower limit of about 0.72 for the P-case and about 0.95 for the U-case. If the coefficient series $\left\{a_{n}\right\}$ is convergent, then the recurrence relations (6) to (9) have been shown to be a stable algorithm: When an error (e.g., due to roundoff) was added to the initial two elements $a_{1}$ and $a_{2}$, the following elements obtained from the recurrence algorithm presented a damped deviation along the index sequence.

The divergence of $\left\{a_{n}\right\}$ as a function of $s_{p}$ can be characterized by the radius of convergence $R_{c}$ (within which the Maclaurin power series would be a valid function). Considering the alternating performance of $\left\{a_{n}\right\}$ as shown in Fig, 1, the Cauchy-Hadamard theorem is chosen to determine the radius of convergence [18]: $1 / R_{c}=\limsup _{n \rightarrow \infty}\left|a_{n}\right|^{\frac{1}{n}}$, where "sup" represents the limit superior. The radius of convergence is the same for the radial ranges of $s>s_{p}$ and $s<s_{p}$ as can be seen from relations (10). Considering that an infinite index is not approachable in a real computation, a truncated radius of convergence $R_{c}^{*}$ is defined as:

$$
\frac{1}{R_{c}^{*}}=\sup \left\{\left|a_{n}\right|^{\frac{1}{n}}: 20 \leqslant n \leqslant 25\right\},
$$

which is a truncated approximation using a finite integer instead of infinity. In Fig. 2 both the P-case (solid line) and the U-case (dash-dotted line) show a decreasing truncated radius of convergence towards zero when $s_{p}$ approaches zero, suggesting that the Maclaurin approximation is not a reliable

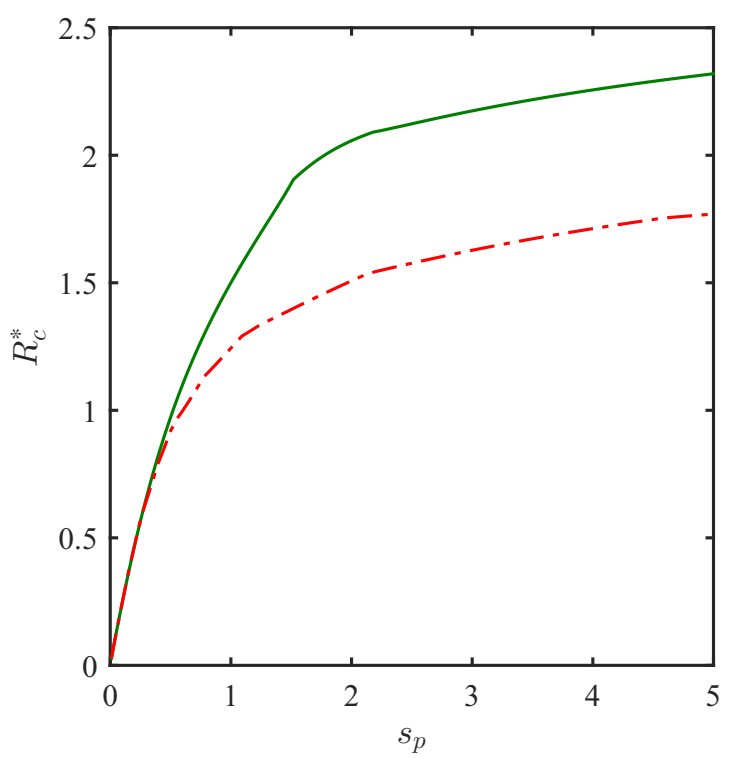

FIG. 2. (Color online) Truncated radius of convergence $R_{c}^{*}$ for the P-case (solid line) and U-case (dash-dotted line) as a function of $s_{p}$. method for solving the T-L integral equation (3) for small values of $s_{p}$, where $\left\{a_{n}\right\}$ could become highly divergent. A smaller lower limit of $s_{p} \sim 0.72$ for the P-case compared to $s_{p} \sim 0.95$ for the U-case is consistent with the performance of the $R_{c}^{*}\left(s_{p}\right)$ curves where the P-case curve is higher than the $\mathrm{U}$-case and hence has a broader applicable range of $s_{p}$.

Additionally, the lower limit of $s_{p}$ for the convergence of $\left\{a_{n}\right\}$ is also shown to be the limit ensuring a valid solution for the boundary condition (11). This coincidence suggests that the validity of a Maclaurin approximant depends on the convergence of its coefficient series $\left\{a_{n}\right\}$, which is consistent with the following: For the P-case, when $s_{p}=0.72$ a solution of $\rho_{b} \sim 1$ is yielded at the outer boundary which is close to the respective radius of convergence of $R_{c}^{*} \sim 1.2$ identified from Fig. 2 and for the U-case a similar phenomenon is observed. It will be shown in Sec. $\mathrm{V}$ that the annular ratio of inner radius to outer radius $R_{\mathrm{io}}=\frac{r_{a}}{r_{b}}=\frac{s_{a}}{s_{b}}$ has a positive correlation with $s_{p}$, hence the Maclaurin approximant should be used for annular geometries with relatively large inner radii. It should be noted that the Maclaurin approximant is convergent and valid for the cylindrical geometry (i.e., a zero inner radius) and for the plane-parallel geometry, both cases having no even-index terms in the power series [1]. The recurrence relations for the cylindrical and plane-parallel plasmas are also given in the Appendix A for reference.

\section{PADÉ RATIONAL APPROXIMANT}

In order to solve annular geometries with small inner radii, the Padé rational approximant is introduced to extrapolate the limit determined by the Maclaurin approximant. It uses a rational function, whose numerator and denominator can be directly calculated from the pre-calculated Maclaurin approximant [14,15], to approximate an underlying solution. The genuine advantage of the Padé approximant, over most other approximation methods, is that it can still work even if the Maclaurin series is divergent. A general analysis to evaluate the validity of a Padé approximant is a complex task, especially for the present case where the mathematics of the T-L integral equation (3) has not been fully solved. Here we verify the validity of the Padé approximant by substituting the obtained solution curve $x(\rho)$ into the T-L integral equation and by checking the error magnitude (as shown in Sec. V).

The Padé approximant has the following form:

$$
[M / N]=\frac{\sum_{i=0}^{L} p_{i} \rho^{i}}{1+\sum_{i=1}^{M} q_{i} \rho^{i}},
$$

where a diagonal rational approximation, i.e., $M=N$, is considered for the present study. Its denominator coefficients $\left\{q_{i}\right\}$ are calculated from the coefficient series $\left\{a_{n}\right\}$ of the Maclaurin approximant following the formulas below, which can be found in the literature about Padé approximant $[14,15,19]$ :

$$
\left[\begin{array}{cccc}
a_{N} & a_{N-1} & \cdots & a_{1} \\
a_{N+1} & a_{N} & \cdots & a_{2} \\
\vdots & \vdots & \ddots & \vdots \\
a_{2 N-1} & a_{2 N-2} & \cdots & a_{N}
\end{array}\right]\left[\begin{array}{c}
q_{1} \\
q_{2} \\
\vdots \\
q_{N}
\end{array}\right]=-\left[\begin{array}{c}
a_{N+1} \\
a_{N+2} \\
\vdots \\
a_{2 N}
\end{array}\right]
$$


and the numerator coefficients $\left\{p_{i}\right\}$ :

$$
p_{i}=\sum_{k=0}^{i} q_{k} a_{i-k}
$$

where $q_{0}=1$ and $p_{0}=a_{0}=0$. The denominator and numerator coefficients for the radial range of $s>s_{p},\left\{q_{i}^{+}\right\}$and $\left\{p_{i}^{+}\right\}$, are calculated from $\left\{a_{i}^{+}\right\}$, and those $\left(\left\{q_{i}^{-}\right\}\right.$and $\left.\left\{p_{i}^{-}\right\}\right)$for $s<s_{p}$ from $\left\{a_{i}^{-}\right\}$. In this case the outer and inner boundary locations $\left(s_{b}\right.$ and $s_{a}$ ) of an annular plasma are determined by:

$$
\frac{d x^{ \pm}}{d \rho}=\frac{\left(\sum_{i=1}^{L} i p_{i}^{ \pm} \rho^{i-1}\right)\left(1+\sum_{i=1}^{M} q_{i}^{ \pm} \rho^{i}\right)-\left(\sum_{i=0}^{L} p_{i}^{ \pm} \rho^{i}\right)\left(\sum_{i=1}^{M} i q_{i}^{ \pm} \rho^{i-1}\right)}{\left(1+\sum_{i=1}^{M} q_{i}^{ \pm} \rho^{i}\right)^{2}}=0
$$

The reliability of a Padé approximant is determined by the accuracy of linear algebraic equation (14). There are a number of algorithms designed to calculate the Padé approximant, depending on the preferred criteria (e.g., reliability or efficiency) and among all, the direct routine of "matrix inversion" is the most stable and reliable method though at the cost of some efficiency [14,19]. The validity of the matrix inversion method is further enhanced by the fact that a low-order Padé rational function is normally chosen for calculation as an excessively high order sometimes causes computational instabilities accumulated from both roundoff and truncation errors [15], especially when a singular matrix [a] occurs in Eq. (14). Morris [19] suggested a general order principle of $N \lesssim 10$ for single-precision computing and hence $N \lesssim 20$ for double precision which is defaulted for the present study. Considering the possible degeneration of the parametric matrix [a] which is likely the case for small values of $s_{p}$, the LU decomposition is used to solve the algebraic equation (14) and an iterative improvement is also supplemented to refine the solution $[15,19]$. An order of 7 to 12 is suggested for the diagonal Padé approximant in this paper, and their stability has been verified: When a small error was added to the first few coefficients of Maclaurin series $\left(\left\{a_{n}\right\}\right)$, the related Padé coefficients showed a negligible magnitude of deviation.

To show the advantage of the Padé approximant for small values of $s_{p}$, i.e., annular geometries with small inner radii, a value of $s_{p}=0.4$ for the P-case is used as an example where the Maclaurin approximant is divergent as shown in Fig. 1. The solution curve $x(\rho)$ obtained from a Padé approximant [9/9] is given for the outer radial range of $s>s_{p}$ and it is shown in Fig. 3(a) as the solid line [the boundary value of $\rho_{b} \sim 1$ is determined by Eq. (16)]. Substituting the calculated $x(\rho)$ and $\frac{d x}{d \rho}$ into the T-L integral equation (3) yields a residual value, and a relative error is defined to reflect the deviation of the approximant solution using:

$\Lambda(\rho)=\left|\frac{-\left(s_{p}+x^{*}\right) \mathrm{e}^{-\rho^{2}} \pm \int_{0}^{\frac{\pi}{2}}\left(s_{p}+x_{z}^{*}\right) e^{-c \rho_{z}^{2}} \frac{d x_{z}^{*}}{d \rho_{z}} d \theta}{-\left(s_{p}+x^{*}\right) e^{-\rho^{2}}}\right|$,

where the superscript " $*$ " refers to the computational results. The validity of the Padé approximant is verified by the error curve $\Lambda(\rho)$ represented by the solid line in Fig. 3(b) which is within the magnitude of $10^{-4}$. The solution curve $x(\rho)$ obtained from the Maclaurin approximant (dash-dotted line), which uses the first 25 elements (excluding $a_{0}=0$ ) in the power series, is also shown in Fig. 3(a) as a comparison and it presents a clear discrepancy from the Padé approximant result beyond $\rho \sim 0.7$. The Maclaurin approximant is not a valid method for solving the T-L integral equation in this case as a divergent $\Lambda(\rho)$ curve is shown in Fig. 3(b), with an error value of $100 \%$ at $\rho \sim 0.8$. When $s_{p}$ is set to be a large value (e.g., $s_{p}=1.6$ ), the Padé and Maclaurin approximants give consistent solution curves and both of their $\Lambda(\rho)$ curves approach zero.

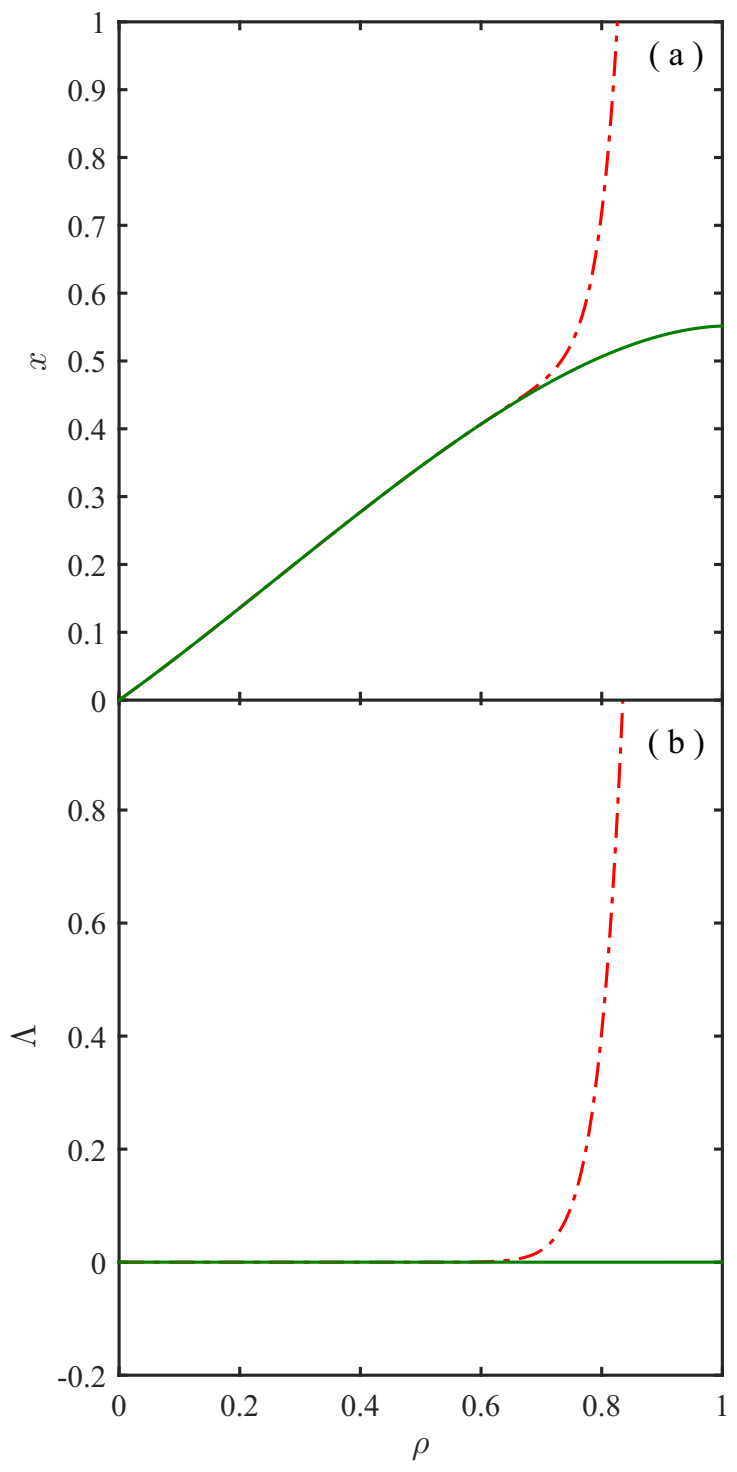

FIG. 3. (Color online) For a P-case annular plasma with $s_{p}=$ 0.4 , (a) solution curve $x(\rho)$, and (b) relative error curve $\Lambda(\rho)$ in the outer radial range $\left(s>s_{p}\right)$ calculated by the Maclaurin approximant (dash-dotted line) and the Padé approximant (solid line). 


\section{ANNULAR LIMITS}

The T-L integral equation (3) can be rewritten as:

$$
\begin{aligned}
& s_{p}\left[-\mathrm{e}^{-\rho^{2}} \pm \int_{0}^{\frac{\pi}{2}} \mathrm{e}^{-c \rho_{z}^{2}} \frac{\mathrm{d} x_{z}}{\mathrm{~d} \rho_{z}} \mathrm{~d} \theta\right] \\
& +\left[-x \mathrm{e}^{-\rho^{2}} \pm \int_{0}^{\frac{\pi}{2}} x_{z} \mathrm{e}^{-c \rho_{z}^{2}} \frac{d x_{z}}{d \rho_{z}} d \theta\right]=0 .
\end{aligned}
$$

On the left-hand side, the expression in the first square brackets has the same form for a plane-parallel plasma, and the expression in the second brackets for a cylindrical plasma. It is interesting to notice that the equation for an annular plasma is a combination of a weighted plane-parallel part (by a factor of $s_{p}$ ) and a cylindrical part. Hence what would be expected is that the annular solution approaches the cylindrical case for small values of $s_{p}$, and when $s_{p}$ is large it should be close to the planeparallel case, indicating that the annular radio (of inner radius to outer radius) $R_{\mathrm{io}}=\frac{s_{a}}{s_{b}}$ should be an increasing function of $s_{p}$ which is verified by the results shown on Figs. 4 and 5 .

The $R_{\text {io }}\left(s_{p}\right)$ curve for the P-case, calculated by the first 25 elements of the Maclaurin series approximant (excluding $a_{0}=0$ ), is shown as the dash-dotted line in Fig. 4(a). It has a lower limit of $s_{p}$ at 0.75 determined by the relation $\sup \left\{\Lambda(\rho): s_{a}<s(\rho)<s_{b}\right\}<10^{-3}$ which states that for each value of $s_{p}$ on the curve, its respective solution curve $x(\rho)$ to the T-L integral equation (3) has a maximum relative error smaller than $10^{-3}$ across the annulus. Hence the calculated $R_{\mathrm{io}}\left(s_{p}\right)$ curve satisfies a well-defined accuracy. Figure 4(b) shows the results from the Padé approximant with a diagonal order of [9/9] to [12/12] represented by the solid line, which has the same solution accuracy of a maximum relative error being less than $10^{-3}$ for the T-L integral equation. The $R_{\mathrm{io}}\left(s_{p}\right)$ curves calculated by both approximants present quite consistent results for the range of $s_{p}>0.75$ (overlapped if plotted on one figure), and the Padé approximant further extrapolates the lower limit of $s_{p}$ from 0.75 determined by the Maclaurin approximant to 0.3 as marked by the vertical dashed lines in Figs. 4(a) and 4(b). Consequently, the lower limit of the annular ratio $R_{\text {io }}$ is extrapolated from 0.35 to 0.1 by the Padé approximant whose range can cover most annular geometries. The $R_{\mathrm{io}}\left(s_{p}\right)$ results for the U-case are plotted in Figs. 5(a) and 5(b) where the Maclaurin and Padé approximants give very consistent results for the range of $s_{p}>1.05$ (overlapped if plotted on one figure), and the latter extrapolates the lower limit of $s_{p}$ from 1.05 to 0.27 , and the limit of $R_{\text {io }}$ from 0.5 to 0.1 . Hence, in summary, the Maclaurin approximant has a valid range of annular ratios of greater than 0.35 for the P-case and greater than 0.5 for the U-case, and the Padé approximant extends the valid ratio range to greater than 0.1 for both the $\mathrm{P}$ and U-cases.

The results in Figs. 4(b) and 5(b) show that the annular ratio $R_{\mathrm{io}}$ and the dimensionless peak position $s_{p}$ have a positive injection relation and hence they are equivalent representations. $R_{\text {io }}$ approaches zero (i.e., cylindrical case) when $s_{p}$ has a small value and it is close to unity (i.e., plane-parallel geometry) when $s_{p}$ becomes large, consistent with the qualitative conclusion deduced from equation (18). Though the Padé approximant can be successfully applied to

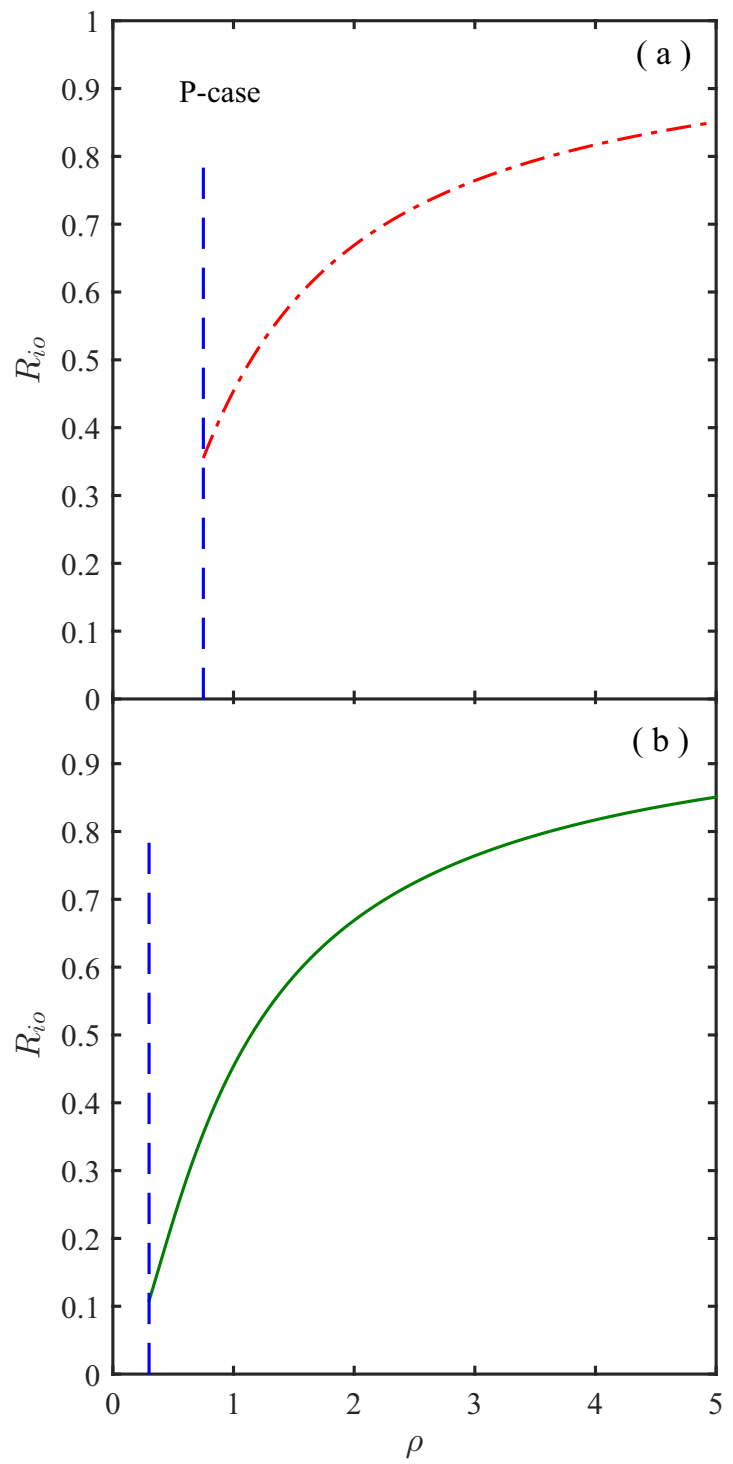

FIG. 4. (Color online) For the P-case plasma, annular ratio $R_{\text {io }}$ of inner radius to outer radius as a function of $s_{p}$ calculated by (a) the Maclaurin approximant (dash-dotted line) and (b) the Padé approximant (solid line). The vertical dashed lines mark the lower limit of $s_{p}$ for validated solutions of the two approximants (followed by Fig. 5).

most annular geometries, there is still a small gap between the annular geometries of $R_{\text {io }} \sim 0.1$ to 0 being unfilled [Figs. 4(b) and 5(b)] where the Maclaurin coefficient series $\left\{a_{n}\right\}$ becomes highly divergent and the Padé approximant calculated from these coefficients also loses validity.

\section{MODELLING RESULTS}

The solution curve $x(\rho)$ to the T-L integral equation, obtained from the Maclaurin approximant (Sec. III) and Padé approximant (Sec. IV), is used to investigate the radial transport of charged particles in collisionless annular plasmas. Radial profiles, for which the radial position is represented by $s=x+s_{p}$ as given in Sec. II, of the normalized plasma density and mean drift velocity of ions (electrons have been 


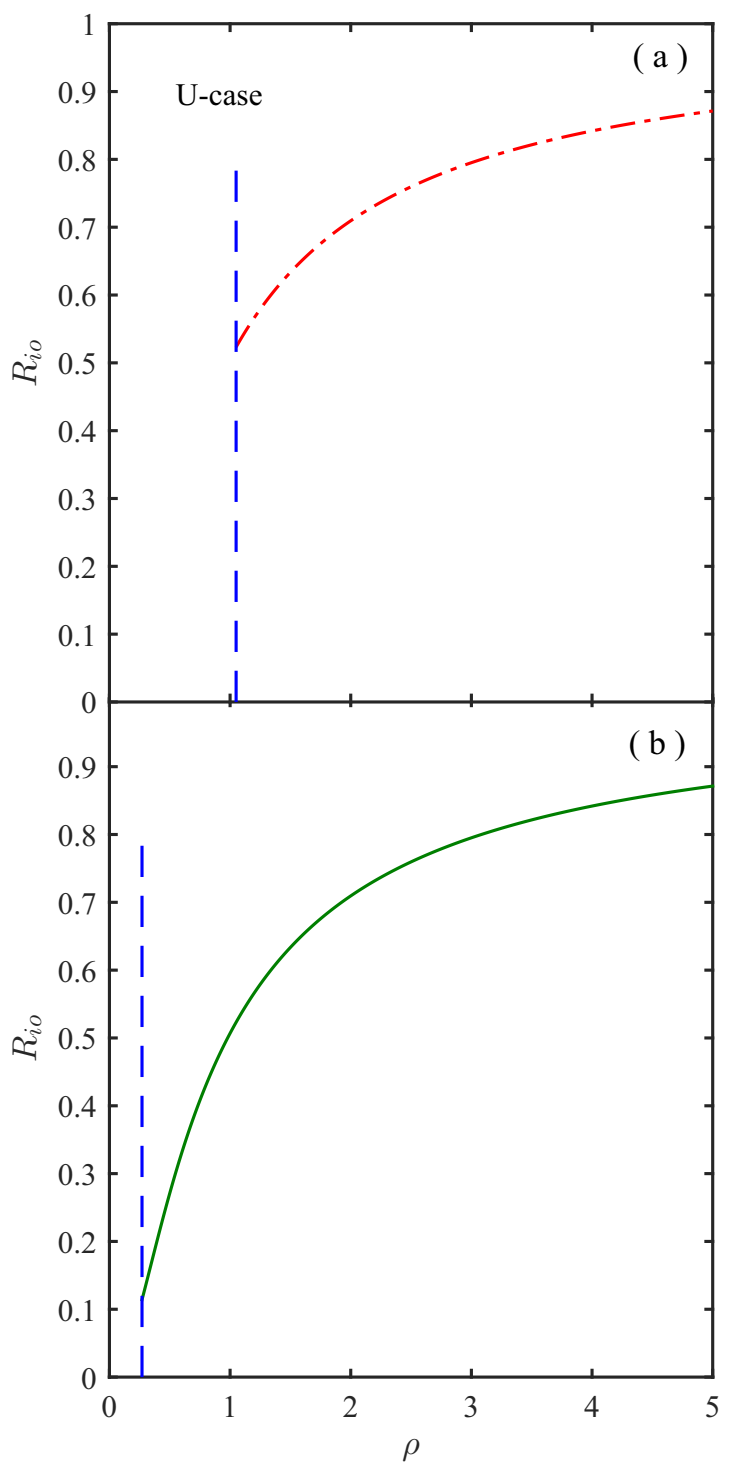

FIG. 5. (Color online) For the U-case plasma, annular ratio $R_{\text {io }}$ as a function of $s_{p}$ calculated by (a) the Maclaurin approximant (dash-dotted line) and (b) the Padé approximant (solid line).

assumed to be in equilibrium) are carefully characterized, and their values are calculated as follows: The former is determined by the Boltzmann relation $\hat{n}=e^{-\eta}$, where $\eta=\rho^{2}$, and the latter, normalized by the Bohm velocity $u_{B}=\sqrt{\frac{e T_{e}}{m_{i}}}$, is given by:

$$
\begin{aligned}
\hat{u} & =\frac{ \pm \sqrt{2} \int_{s_{p}}^{s} s_{z} e^{-c \eta_{z}} d s_{z}}{\int_{s_{p}}^{s} s_{z} e^{-c \eta_{z}}\left(\eta-\eta_{z}\right)^{-\frac{1}{2}} d s_{z}} \\
& =\frac{ \pm \sqrt{2} \int_{0}^{\frac{\pi}{2}}\left(s_{p}+x_{z}\right) e^{-c \rho_{z}^{2}} \frac{d x_{z}}{d \rho_{z}} \rho \cos \theta d \theta}{\int_{0}^{\frac{\pi}{2}}\left(s_{p}+x_{z}\right) e^{-c \rho_{z}^{2}} \frac{d x_{z}}{d \rho_{z}} d \theta}
\end{aligned}
$$

where the numerator and denominator represent the ion flux and plasma density, respectively. The normalized plasma density and mean drift velocity are only functions of the dimensionless peak position $s_{p}$ or, more practically, the annular ratio $R_{\mathrm{io}}$ which is monotonically connected to $s_{p}$ as shown by Figs. 4 and 5, and hence their radial profiles are general results that are independent of the specific gas type and of the Paschen number (i.e., gas pressure and physical size) of the discharge. The following Sec. IV A shows these general profiles for different annular geometries, and in Sec. IV B, the annular modeling is applied to an argon plasma and the electron temperature is calculated as a function of the Paschen number $P_{\mathrm{as}}=p_{g} r_{b}$, where $p_{g}$ and $r_{b}$ are the gas pressure and the physical outer radius of the annulus, respectively.

\section{A. General radial profiles}

Radial profiles of the normalized plasma density for annular geometries of $R_{\mathrm{io}}=0.2$ to 0.8 are given in Fig. 6(a) for the P-case, with the radial position being normalized by $\hat{r}=\frac{r}{r_{b}}=$

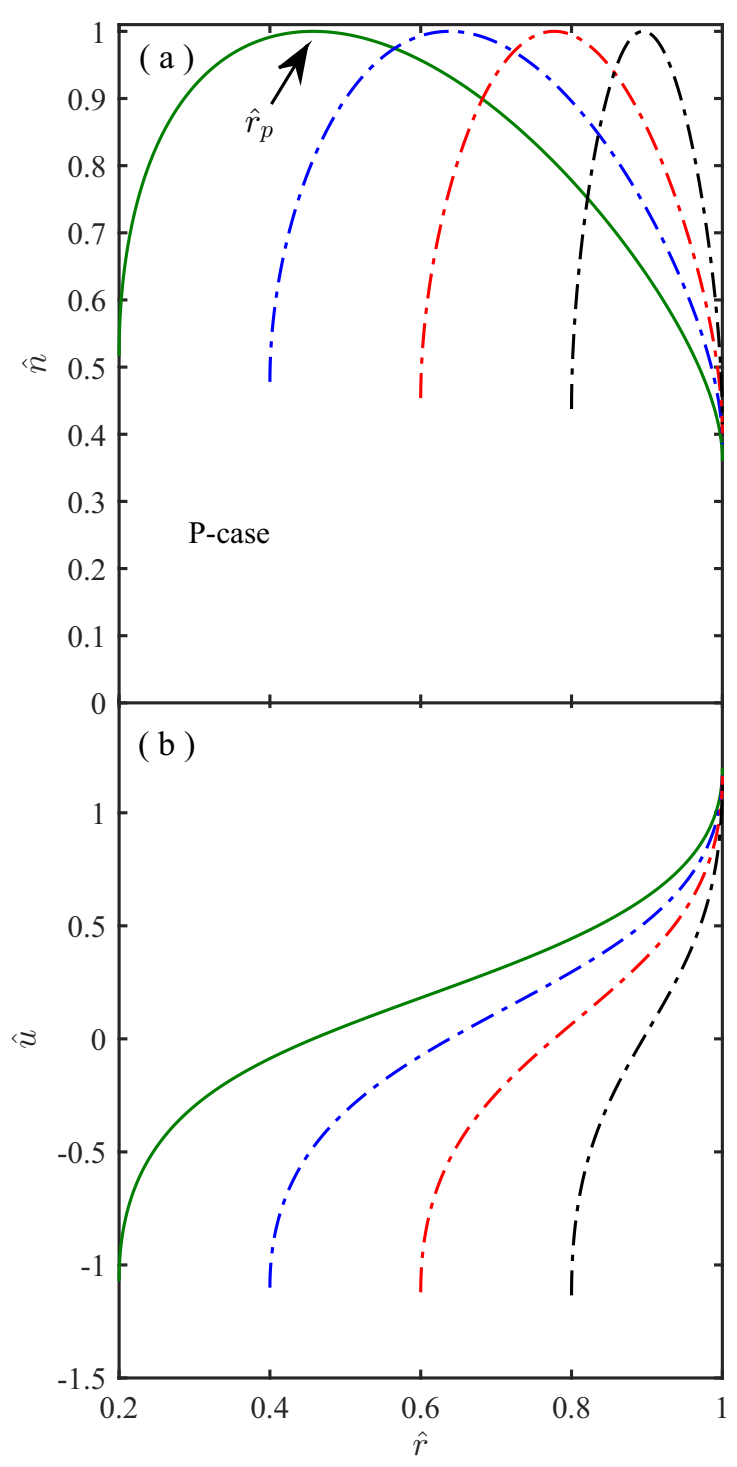

FIG. 6. (Color online) For the P-case plasma, radial profiles of (a) normalized plasma density $\hat{n}$, and (b) normalized mean drift velocity of ions $\hat{u}$ for different annular geometries of $R_{\mathrm{io}}=0.2,0.4,0.6$, and 0.8 . The dash-dotted lines represent overlapped results that can be calculated by both the Maclaurin and Padé approximants, and the solid line represents the result that is only valid using the Padé approximant (followed by Fig. 7). 


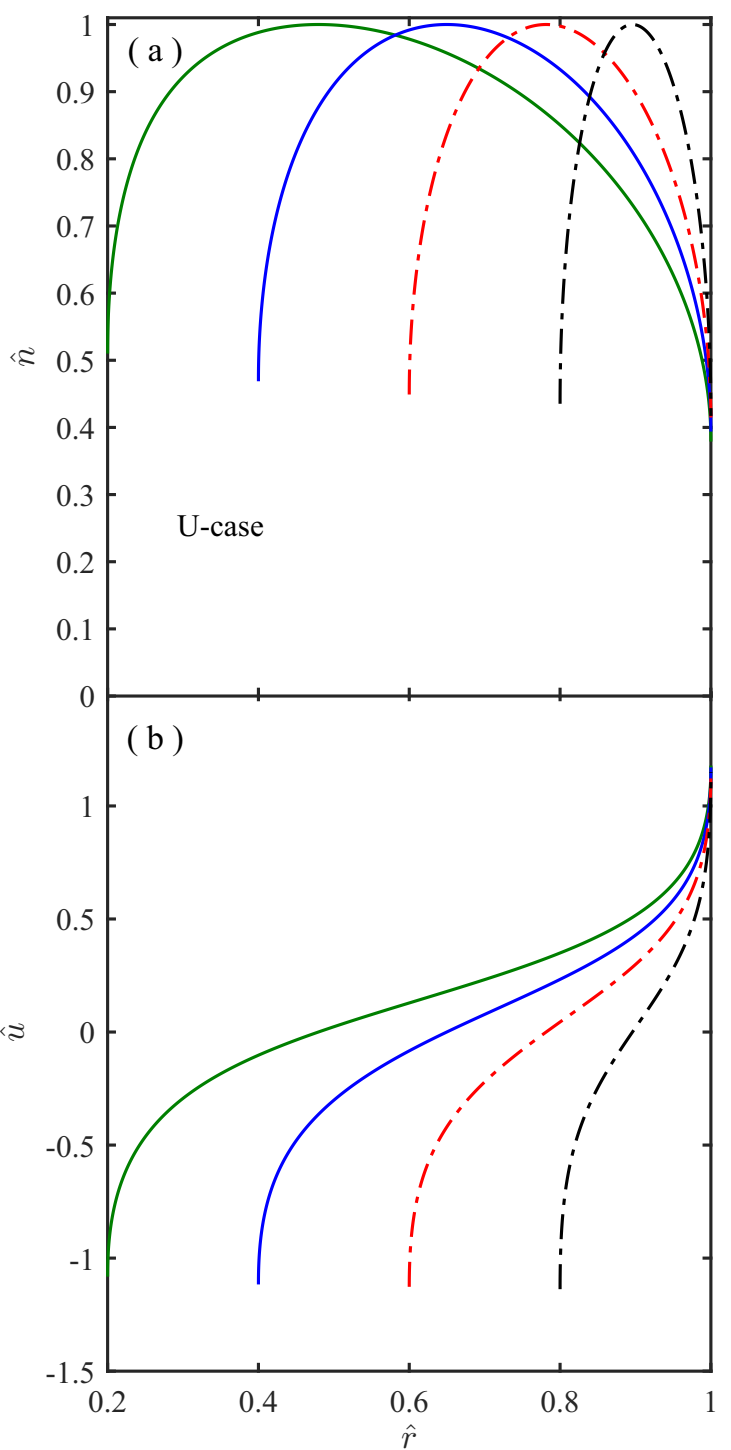

FIG. 7. (Color online) For the U-case plasma, radial profiles of (a) normalized plasma density $\hat{n}$ and (b) normalized mean drift velocity $\hat{u}$ for different annular geometries of $R_{\mathrm{io}}=0.2,0.4,0.6$, and 0.8 .

$\frac{s}{s_{b}}$. The solid line for $R_{\text {io }}=0.2$ is obtained from the Padé approximant where the Maclaurin approximant fails as shown in Fig. 4, while the dash-dotted lines can be obtained from both approximants which give very consistent (overlapped) results. The boundary densities at the inner and outer sides are about 0.35 to 0.5 of the maximum density with the inner-side value being slightly higher than the outer. The peak position of the density profile is closer to the inner boundary due to a larger loss area at the outer side. The density profile becomes more symmetric as the annular ratio $R_{\text {io }}$ increases from 0.2 to 0.8 (approaching the plane-parallel case). The mean drift velocities at the inner and outer boundaries, as shown in Fig. 6(b), have a value of $\sim 1.15$ times the Bohm velocity as a result of the infinite-field boundary conditions governed by Eqs. (11) or (16).

Figures 7(a) and 7(b) show the results for the U-case where the plasma density and the mean drift velocities present similar behaviors to those of the P-case. By carefully comparing Figs. 6(a) and 7(a), the normalized density is found greater for the U-case by about $1 \%$ to $5 \%$ compared to the P-case for different annular geometries in the radial range of $\hat{r}>\hat{r}_{p}$, and the mean drift velocity thereby, as shown in Figs. 6(b) and 7(b), is greater in the P-case than the U-case due to a larger proportion of high-velocity ions in the plasma flux moving towards the boundary.

\section{B. Electron temperature in argon plasma}

The electron temperature is included in the dimensionless position parameter $s=\frac{r v_{i z}^{(P / U)}}{\sqrt{2} u_{B}}$ where the ion generation rate $v_{i z}^{(P / U)}$ and the Bohm velocity $u_{B}$ are functions of the electron temperature $T_{e}$. For the P-case the ionization (generation) rate is given by $v_{i z}^{(P)}=n_{g} \mu_{i z}$, where $n_{g}$ is the gas density and the rate constant $\mu_{i z}$ over Maxwellian electrons in an argon plasma is given in Appendix B. As for the U-case the value of $v_{i z}^{(U)}$ is the ratio of the ion generation density to the maximum plasma density as stated in Sec. II. However, in the U-case the ion generation rate is determined by the external ion supply or the diffusion from an extra dimension (e.g., axial dimension), and these effects have not been included within the present scope. Hence the results of electron temperature are only given for the P-case which is governed by the self-consistent particle conservation. In order to present the electron temperature as a function of the Paschen number, the position parameter at the outer boundary which includes the physical dimension the discharge is first given by:

$$
s_{b}=\frac{r_{b} v_{i z}}{\sqrt{2} u_{B}}=\frac{\mu_{i z}}{\sqrt{2} u_{B}} \frac{P_{\mathrm{as}}}{e T_{g}},
$$

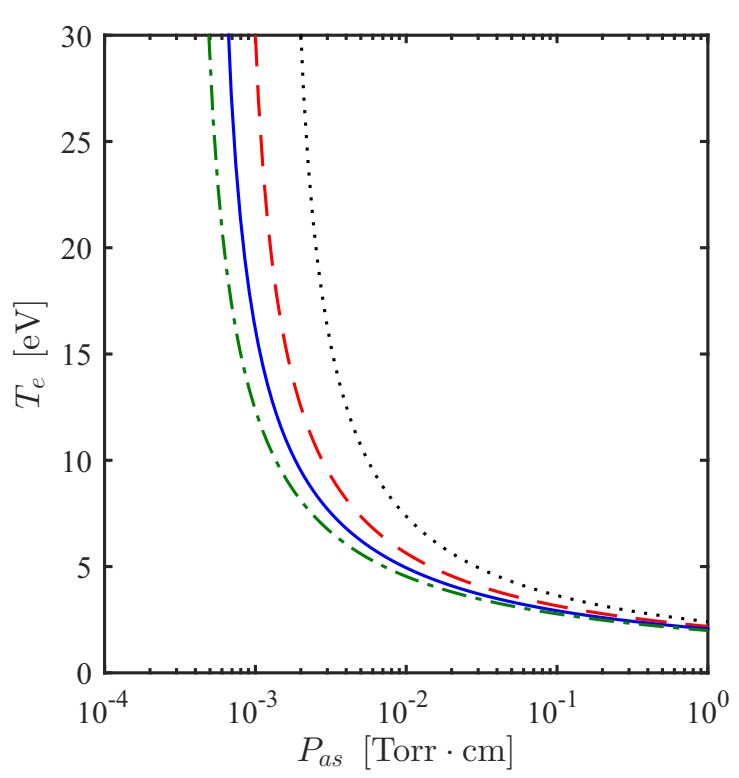

FIG. 8. (Color online) Electron temperature $T_{e}$ as a function of the Paschen number $P_{\text {as }}$ for the P-case argon plasmas (with $s_{b}$ calculated by the Padé approximant) for different annular geometries of $R_{\mathrm{io}}=0.2$ (dash-dotted line), 0.4 (solid line), 0.6 (dashed line), and 0.8 (dotted line). 
whose value can be calculated from the boundary conditions (11) or (16).

Here we present the results of electron temperature in a lowpressure (collisionless) argon plasma. The input parameters are the Paschen number and the annular ratio which determines the value of $s_{b}$, and the output parameter is the electron temperature $T_{e}$ by solving the above equation (20). The argon has a atomic mass of $m_{g}=39.95 \mathrm{u}\left(\mathrm{u}=1.6605 \times 10^{-27} \mathrm{~kg}\right.$ is the atomic mass unit) and a room temperature of $T_{g}=0.026 \mathrm{~V}$ is used for the neutral argon gas. The electron temperature for which $s_{b}$ is calculated using the Padé approximant, is plotted in Fig. 8 as a function of the Paschen number ranging from $10^{-4}$ to 1 Torr $\mathrm{cm}$ for different annular geometries of $R_{\mathrm{io}}=0.2$ to 0.8 . The $T_{e}\left(P_{\mathrm{as}}\right)$ curve has a higher level for larger annular ratios and the difference is more dramatic in the low-Paschen-number range. For the high Paschen numbers the electron temperatures of different annular ratios converge to a similar value of $\sim 2.4 \mathrm{eV}$ at $P_{\mathrm{as}}=1$.

\section{CONCLUSION}

In this paper, the Maclaurin and Padé approximants are used to solve the Tonks-Langmuir theory for an annular plasma in the P-case or U-case. The coefficient series of the Maclaurin approximant is represented in forms of recurrence relations which are convenient for computation. This approximant method has been shown to have a lower limit for the annular ratio, below which it becomes divergent and invalid. The Padé approximant is consequently introduced and shown to be a more robust method. Though its numerator and denominator are calculated using the coefficients of the Maclaurin approximant, it extrapolates the annular ratio limit to a broader range which can include annular geometries with small inner radii and cover most annular geometries. However, there is still a small gap between the annular ratio of 0.1 to 0 (cylindrical geometry) where the coefficient series of the Maclaurin approximant is highly divergent and the Padé approximant also loses its validity. The validity of the two approximants is tested by substituting their solution curves into the T-L integral equation and by checking the magnitude of the error.

The annular modeling is applied to different annular geometries and obtains general radial profiles of the normalized plasma density and mean drift velocity of ions across the annulus. Both profiles are independent of the gas type and the Paschen number of the discharge, and they are asymmetric about the maximum density position and present similar performance for the P-case and U-case. The electron temperature is calculated in argon for the $\mathrm{P}$-case as a function of the Paschen number and shows positive correlation with the annular ratio as enhanced ionization is needed to balance an increased ion loss at the boundaries.

\section{ACKNOWLEDGMENT}

We thank Professor Robert Dewar for discussions on Padé approximant.

\section{APPENDIX A: RECURRENCE RELATIONS FOR CYLINDRICAL AND PLANE-PARALLEL PLASMAS}

For a cylindrical plasma the even-index coefficient terms $\left\{a_{2 n}\right\}$ of the Maclaurin series (4) are zero, and the odd-index terms $\left\{a_{2 n+1}\right\}$, where $a_{1}=1$ are given in forms of recurrence relations for the $\mathrm{P}$-case:

$$
\begin{aligned}
a_{2 n+1}= & \frac{1}{2(n+1)-\frac{(2 n+1) ! !}{(2 n) ! !}}\left\{\left[\frac{(2 n+1) ! !}{(2 n) ! !}-1\right]\right. \\
& \times \sum_{i=1}^{n} \frac{(-1)^{n+1-i}}{(n+1-i) !} a_{2 i-1}-\sum_{i=2}^{2 n} i a_{i} \\
& \left.\times \sum_{j=1}^{n+2-\left\lceil\frac{i+1}{2}\right\rceil} \frac{(-1)^{n+2-\left\lceil\frac{i+1}{2}\right\rceil-j}}{\left(n+2-\left\lceil\frac{i+1}{2}\right\rceil-j\right) !} a_{2 j+2\left\lceil\frac{i-1}{2}\right\rceil-i}\right\}
\end{aligned}
$$

and the U-case:

$$
\begin{aligned}
a_{2 n+1}= & \frac{1}{2(n+1)-\frac{(2 n+1) ! !}{(2 n) ! !}}\left\{\frac{(2 n+1) ! !}{(2 n) ! !} \sum_{i=1}^{n} \frac{(-1)^{n+1-i}}{(n+1-i) !}\right. \\
& \left.\times a_{2 i-1}-\sum_{i=2}^{2 n} i a_{i} a_{2 n+2-i}\right\} .
\end{aligned}
$$

Similarly, for a plane-parallel plasma the even-index terms $\left\{a_{2 n}\right\}$ are zero, and the odd-index terms $\left\{a_{2 n+1}\right\}\left(a_{1}=\frac{2}{\pi}\right)$ are given for the $\mathrm{P}$-case:

$$
\begin{aligned}
a_{2 n+1}= & \frac{1}{(2 n+1)}\left\{\frac{(-2)^{n}}{(2 n-1) ! !} \frac{2}{\pi}\right. \\
& \left.+\sum_{i=1}^{n} \frac{(-1)^{n-i}}{(n-i+1) !}(2 i-1) a_{2 i-1}\right\}
\end{aligned}
$$

and the U-case:

$$
a_{2 n+1}=\frac{(-2)^{n}}{(2 n+1) ! !} \frac{2}{\pi}
$$

\section{APPENDIX B: IONIZATION RATE CONSTANT FOR MAXWELLIAN ELECTRONS IN ARGON PLASMA}

The rate constant used for the argon ionization calculation in Eq. (20) is given by:

$$
\mu_{i z}=\left(\frac{8 e}{\pi m T_{e}^{3}}\right)^{1 / 2} \int_{0}^{\infty} \sigma_{i z}\left(\epsilon_{e}\right) e^{-\frac{\epsilon_{e}}{T_{e}}} \epsilon_{e} d \epsilon_{e}
$$

where $\epsilon_{e}$ is the electron energy and $\sigma_{i z}$ is the ionization cross section for which Phelps's formula [20] is used:

$$
\sigma_{i z}=\frac{970}{(\epsilon+70)^{2}}(\epsilon-15.8)+0.06 \mathrm{e}^{-\frac{\epsilon}{9}}(\epsilon-15.8)^{2},
$$

where $\sigma_{i z}$ is in the unit of $10^{-20} \mathrm{~m}^{2}$. 
[1] L. Tonks and I. Langmuir, Phys. Rev. 34, 876 (1929).

[2] M. A. Lieberman and A. J. Lichtenberg, Principles of Plasma Discharges and Materials Processing, 2nd ed. (John Wiley \& Sons, New York, 2005).

[3] P. Chabert and N. Braithwaite, Physics of RadioFrequency Plasmas (Cambridge University Press, Cambridge, 2011).

[4] W. Schottky, Phys. Z. 25, 635 (1924).

[5] V. A. Godyak, Soviet Radio Frequency Discharge Research (Delphic Associates, Falls Church, VA, 1986).

[6] E. R. Harrison and W. B. Thompson, Proc. Phys. Soc. 74, 145 (1959).

[7] J. F. Waymouth, Phys. Fluids 7, 1843 (1964).

[8] G. Cunge et al., J. Appl. Phys. 89, 3580 (2001).

[9] V. N. Volynets et al., J. Appl. Phys. 99, 043302 (2006).

[10] Y. Zhang, C. Charles, and R. Boswell, Phys. Plasmas 21, 063511 (2014).

[11] Y. Zhang, C. Charles, and R. Boswell, Phys. Plasmas 22, 073510 (2015).
[12] J. H. Ingold, A. Garscadden, and L. M. Chanin, in Gaseous Electronics, Vol. 1, edited by M. N. Hirsh and H. J. Oskam (Academic Press, New York, 1978), Chap. 2, p. 19.

[13] L. M. Biberman, V. S. Vorob'ev, and I. T. Yakubov, Kinetics of Nonequilibrium Low-Temperature Plasmas (Consultants Bureau, New York, 1987).

[14] G. A. Baker and P. Graves-Morris, Padé Approximants (Cambridge University Press, New York, 1996).

[15] W. H. Press et al., Numerical Recipes: The Art of Scientific Computing, 3rd ed. (Cambridge University Press, New York, 2007).

[16] M. A. Lieberman and C. Charles, Phys. Rev. Lett. 97, 045003 (2006).

[17] G. H. Hardy, A Course of Pure Mathematics, 10th ed. (Cambridge University Press, Cambridge, 1967).

[18] S. Lang, Complex Analysis, 4th ed. (Springer, New York, 1999).

[19] P. Graves-Morris, in Padé Approximation and its Applications, Lecture Notes in Mathematics, Vol. 765, edited by L. Wuytack (Springer, Berlin, 1979), p. 231.

[20] A. V. Phelps and Z. L. Petrovic, Plasma Sources Sci. Technol. 8, R21 (1999). 